\title{
Image guidance in transdiscal fixation for high-grade spondylolisthesis in adults with correct spinal balance
}

\author{
Juan Delgado-Fernández, MD, Paloma Pulido, MD, PhD, María Ángeles García-Pallero, MD, \\ Guillermo Blasco, MD, Natalia Frade-Porto, MD, and Rafael G. Sola, MD, PhD \\ Division of Neurosurgery, Department of Surgery, University Hospital La Princesa, Madrid, Spain
}

\begin{abstract}
OBJECTIVE Spondylolisthesis is a prevalent spine disease that recent studies estimate could be detected in $9 \%$ of the population. High-grade spondylolisthesis (HGS), however, is much less frequent, which makes it difficult to develop a general recommendation for its treatment. Posterior transdiscal fixation was proposed in 1994 for HGS, and the use of spine navigation could make this technique more accessible and reduce the morbidity associated with the procedure. The purpose of this study was to present a case series involving adult patients with HGS and correct spinal alignment who were treated with transdiscal pedicle screw placement guided with neuronavigation and compare the results to those achieved previously without image guidance.

METHODS The authors reviewed all cases in which adult patients with correct spinal alignment were treated for HGS with posterior transdiscal instrumentation placement guided with navigation between 2014 and 2016 at their institution. The authors compared preoperative and postoperative spinopelvic parameters on standing radiographs as well as Oswestry Disability Index (ODI) scores and visual analog scale (VAS) scores for low-back pain. Follow-up CT and MRI studies and postoperative radiographs were evaluated to identify any screw malplacement or instrumentation failure. Any other intraoperative or postoperative complications were also recorded.
\end{abstract}

RESULTS Eight patients underwent posterior transdiscal navigated instrumentation placement during this period, with a mean duration of follow-up of 16 months (range 9-24 months). Six of the patients presented with Meyerding grade III spondylolisthesis and 2 with Meyerding grade IV. In 5 cases, L4-S1 instrumentation was placed, while in the other 3 cases, surgery consisted of transdiscal L5-S1 fixation. There was no significant difference between preoperative and postoperative spinopelvic parameters. However, there was a statistically significant improvement in the mean VAS score for low-back pain ( $6.5 \pm 1.5$ vs $4 \pm 1.7$ ) and the mean ODI score ( $49.2 \pm 19.4$ vs $37.7 \pm 22)(p=0.01$ and $p=0.012$, respectively). Six patients reduced their use of pain medication. There were no intraoperative or postoperative complications during the hospital stay, and as of the most recent follow-up, no complications related to pseudarthrosis or hardware failure had been observed.

CONCLUSIONS Treatment with posterior transdiscal pedicle screws with in situ fusion achieved good clinical and radiological outcomes in patients with HGS and good sagittal spinal balance. The use of navigation and image guidance was associated with improved results in this technique, including a reduction in postoperative and intraoperative complications related to screw malplacement, pseudarthrosis, and instrumentation failure.

https://thejns.org/doi/abs/10.3171/2017.10.FOCUS17557

KEY WORDS high-grade spondylolisthesis; transdiscal fixation; spine navigation; image guidance

$\mathrm{S}$ PONDYLOLISTHESIS refers to the anterior or posterior displacement of a vertebral body over the adjacent one. The prevalence of this condition is not well defined. A classic study by Roche and Rowe showed that the prevalence was about $4.2 \%,{ }^{22}$ whereas in recent studies in which spondylolysis was analyzed in CT images obtained in an unselected community sample of adults ${ }^{11}$ or in patients with other pathologies not related to lumbar pain, ${ }^{12}$ a pars articularis defect was detected, respectively, in $9 \%$ and $11.2 \%$ of subjects. However, the incidence of high-grade spondylolisthesis (HGS), defined as slips greater than 50\%-also described as Meyerding grade

ABBREVIATIONS HGS = high-grade spondylolisthesis; ODI = Oswestry Disability Index; VAS = visual analog scale.

SUBMITTED August 29, 2017. ACCEPTED October 31, 2017.

INCLUDE WHEN CITING DOI: 10.3171/2017.10.FOCUS17557. 
III and IV spondylolisthesis-is less frequent, accounting for only $19 \%$ of spondylolisthesis cases..$^{10}$ Nowadays, there is a major consensus about the usefulness of surgery in HGS, although it is more controversial in low-grade cases, ${ }^{2,28}$ but the low incidence of HGS has made it difficult to determine the most useful surgical approach for its treatment. The main objective of surgical treatment of spondylolisthesis is to decompress and fuse the vertebrae, with or without reduction of the slipped vertebra. Many approaches have been described, including anterior and posterior approaches, with or without interbody fusion; posterolateral fusion without decompression; L-5 vertebrectomy (Gaines procedure); and circumferential fusion and reduction. ${ }^{2,16}$ Another of the main controversies related to HGS is the requirement of slippage reduction, which is mainly recommended in cases of spinal imbalance due to the risk of neurological impairment during the procedure. ${ }^{2,14}$ In those patients in whom spinal balance is conserved, most authors agree that slippage reduction can be avoided.2,17 Prior to fixation techniques, posterior in situ fusion was performed, and it was associated with a risk of pseudarthrosis between $17 \%$ and $50 \%$. This risk was reduced with posterior fixation and also with the use of interbody implants and circumferential approaches. ${ }^{4,20}$ In 1994, Abdu and colleagues ${ }^{1}$ proposed a posterior approach with transdiscal pedicle screw fixation from S-1 to L-5, which has been used by different authors and has also been described as "delta" fixation. ${ }^{3-5,8,15,19,23,26}$

More recently, the implementation of 3D image-guidance spine systems has improved the accuracy of instrumentation placement and decreased its morbidity. ${ }^{24,27}$ Also, the acquisition of multiplanar images allows guidance through a complex anatomy while showing the planned trajectory to the surgeon. Moreover, new approaches and techniques are easier thanks to its application. ${ }^{25}$ The objective of our study was to present our case series of HGS treated with transdiscal pedicle screws guided with 3D neuronavigation in adult patients with correct spinal balance and study whether image guidance can improve results compared with previous reports.

\section{Methods}

This study is a retrospective review of HGS cases in which patients underwent surgery with transdiscal L5-S1 fixation between 2014 and 2016 at our institution. Inclusion criteria included correct sagittal balance, vertebral displacement greater than 50\% (Meyerding grade III or IV spondylolisthesis), and chronic low-back pain. Some patients also had lower-extremity symptoms attributable to radiculopathy. Patients with signs of L4-5 instability (ligament hypertrophy, facet joint effusion, reduction in intervertebral distance, and a difference of more than $3 \%$ between flexion and extension standing radiographs) were candidates for fixation from L-4 through S-1. Only cases involving patients older than 18 years (with no upper limit) were included in the study.

We analyzed demographic and clinical parameters, including age, sex, symptoms, preoperative pain level, and daily activities. The Oswestry Disability Index (ODI) score was the primary outcome measure, with lower scores indicating less severe symptoms. ${ }^{7}$ The secondary outcome measure was improvement in low-back pain as measured on a visual analog scale (VAS), with values ranging from 0 to 10 (10 indicating maximal pain). ${ }^{9}$ VAS and ODI scores were obtained before surgery and during follow-up. Postoperative follow-up included, at a minimum, assessment during the 1st month and at 3, 6, and 12 months after surgery.

Preoperative radiological studies included CT and MRI in all cases. In addition, standing anteroposterior and lateral lumbosacral radiographs and lateral flexion-extension images, together with full spine standing lateral radiographs for spinal and pelvic alignment, were obtained in every case. Postoperative imaging studies included CT at 3 months after surgery and MRI at 6 months. Standing radiographs were obtained in the event of complications and during the first follow-up visit, 1 month after surgery. CT and MR images were assessed by an independent radiologist not involved in the surgical procedure. Fusion was evaluated according to the Ray criteria ${ }^{21}$ on CT images.

Grading of slippage was assessed according to the classification of Meyerding. Slip angle was defined according to Dubousset's lumbosacral angle. ${ }^{6}$ Pelvic incidence angle, pelvic tilt, and sacral slope were measured as proposed by Labelle and colleagues. ${ }^{13}$

Surgery was performed on a Jackson radiolucent surgical table with image guidance. A midline incision was made at the level of the spondylolisthesis, and subperiosteal dissection was performed until exposure of transverse process at every level to be treated. Once the reference array was attached to the lower spinous process of the sacrum at S-2 or S-3, images were obtained with intraoperative 3D fluoroscopy (Siemens Medical Solutions) and 3D reconstruction was performed by the Brainlab system for spine navigation. After calibration of the probe, tap, and screwdriver (Fig. 1), the pedicle screws were inserted. The technique of transdiscal fixation has previously been described by Abdu et al. ${ }^{1}$ In our case series, an imageguided drill (Brainlab) was used for initial trajectory planning from the S-1 pedicle, through the endplate of S-1, crossing the intervertebral disc, and reaching the L- 5 body (Fig. 2). Afterward, a previously calibrated screw $(7.5 \mathrm{~mm}$ in diameter and $60-70 \mathrm{~mm}$ in length; Zodiac, Alphatec Spine) was placed with imaging guidance. In this technique, pedicle screws are inserted obliquely through the pedicle of S-1 in an anteromedial direction to the sacral promontory. Then with continued image guidance, using the calibrated screwdriver, the screws are directed across the L5-S1 intervertebral disc to the L-5 body. In the upper levels, screws were also placed with image guidance in a standard fashion. Once the instrumentation was finished, another fluoroscopic 3D scan is performed to check that all screws were correctly positioned (Fig. 3). In patients with radiculopathy, a hemilaminectomy and foraminotomy of the affected root were performed. Finally, in all cases, posterolateral and isthmic fusion with autologous iliac crest bone and heterologous bone graft substitute was performed. After surgery, a lumbosacral orthosis was prescribed for 3 months.

Statistical analysis was performed with IBM SPSS 21.0 (IBM Corp.). Preoperative and postoperative data were 

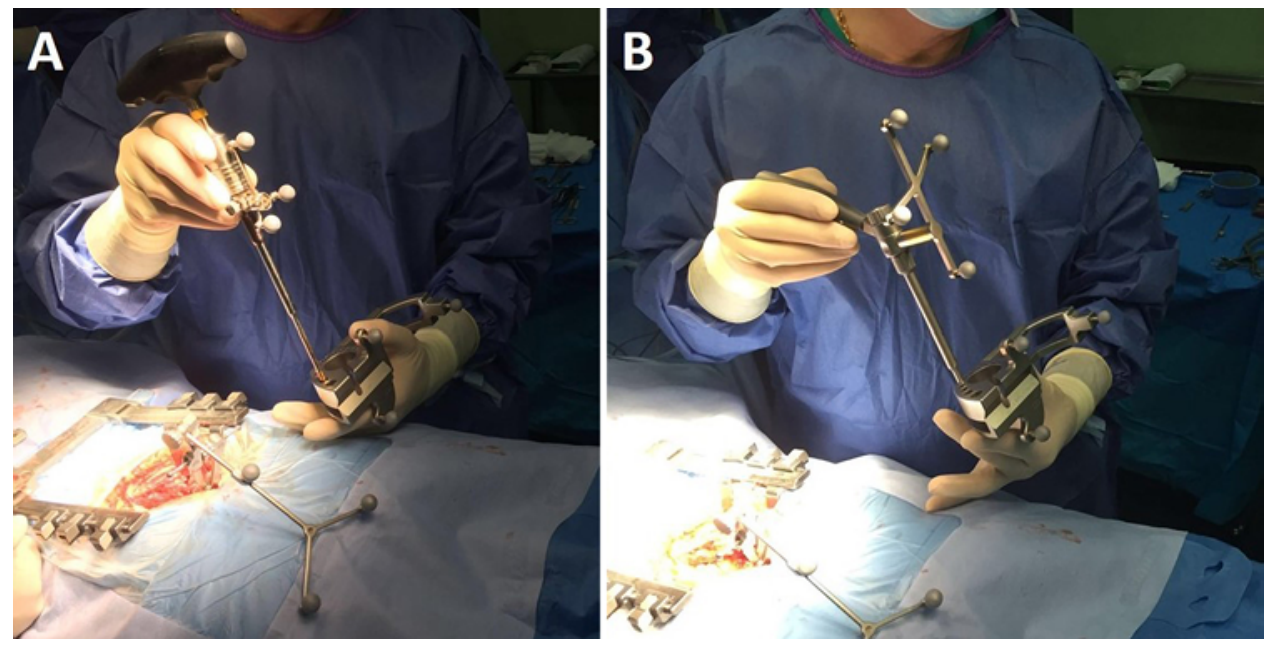

FIG. 1. Intraoperative photographs showing the calibration of the awl-tap (A) and drill guide (B).

compared by means of the Wilcoxon signed-rank test. A $\mathrm{p}$ value $\leq 0.05$ was considered to indicate a statistically significant difference.

Because the study was a retrospective review of patients, no informed consent was required for enrollment.

\section{Results}

During the period analyzed in our study, 8 patients (all female) underwent surgery for HGS. Their mean age was 47.5 years (range 37-62 years). A summary of the patients' spinopelvic parameters is shown in Table 1 . Six patients presented with Meyerding grade III spondylolisthesis and only 2 patients with Meyerding grade IV displacement of L-5 over S-1. In 5 cases, L4-S1 fixation was performed because of signs of L4-5 instability on dynamic radiographs and/or MRI (see Methods); in the remaining 3 cases, the signs of instability were limited to the lumbosacral joint, and only transsacral L5-S1 instrumentation was used. No reduction of the listhesis was performed in any case, as there was no spinal imbalance. The median surgical time
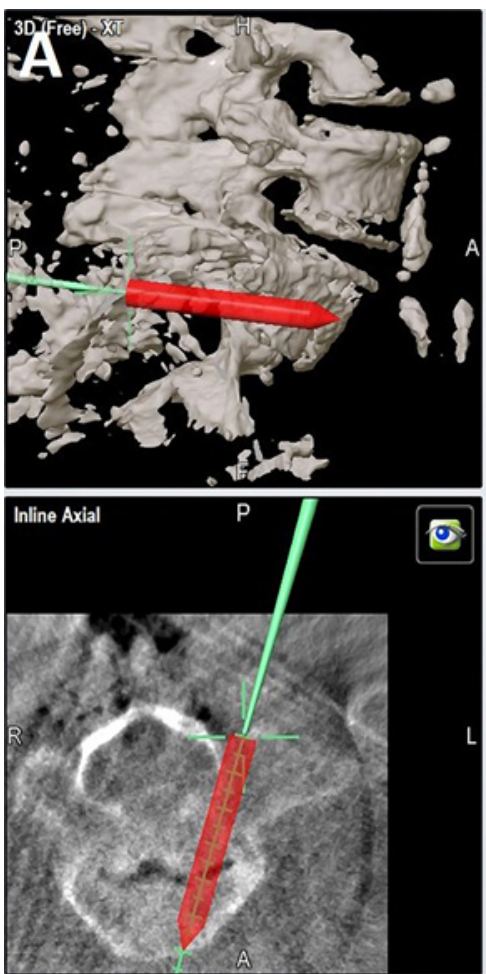
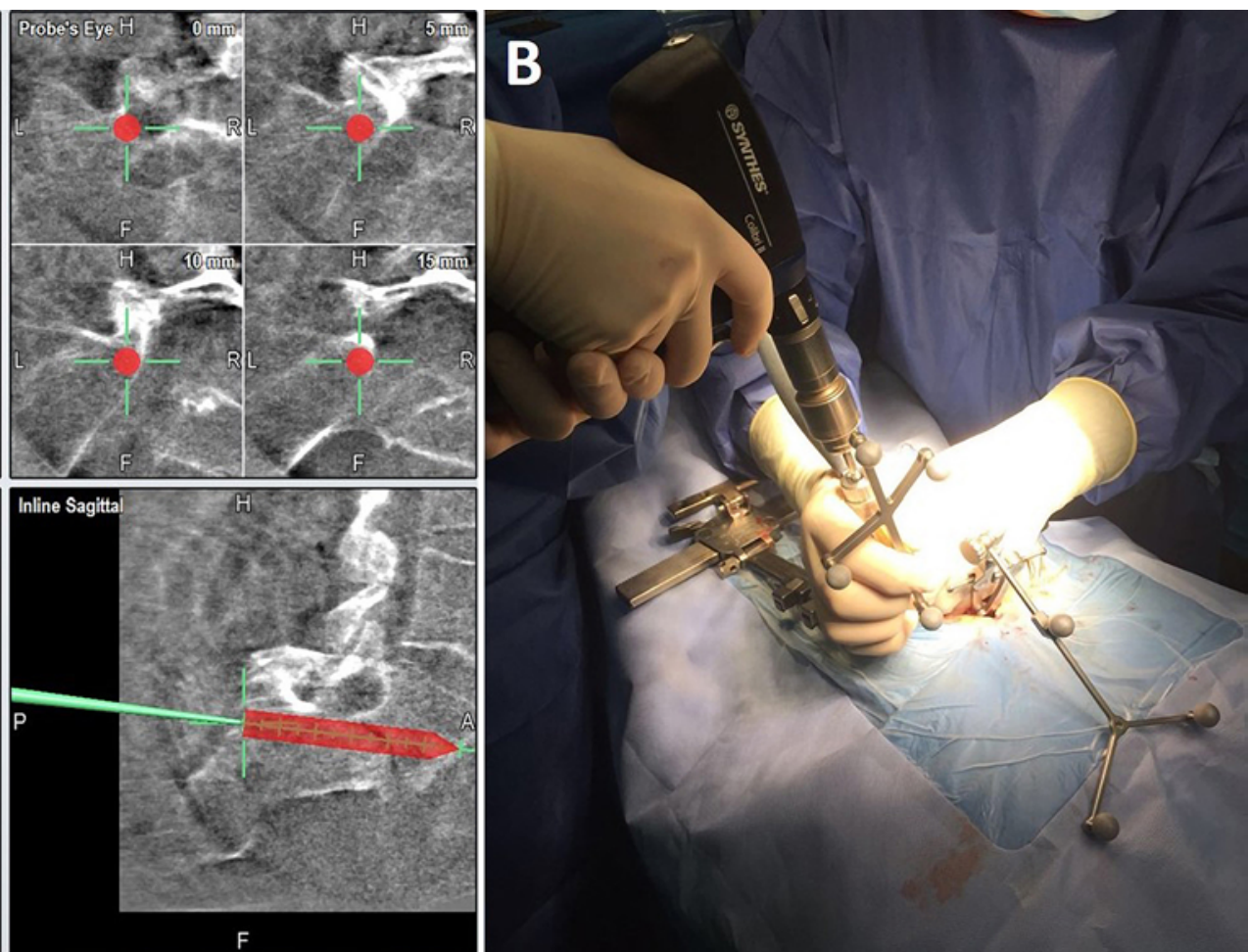

FIG. 2. A: Photograph of the navigation screen showing the correct position of the transdiscal L5-S1 screw. B: Intraoperative photograph of navigation-guided drilling for transdiscal screw placement. 

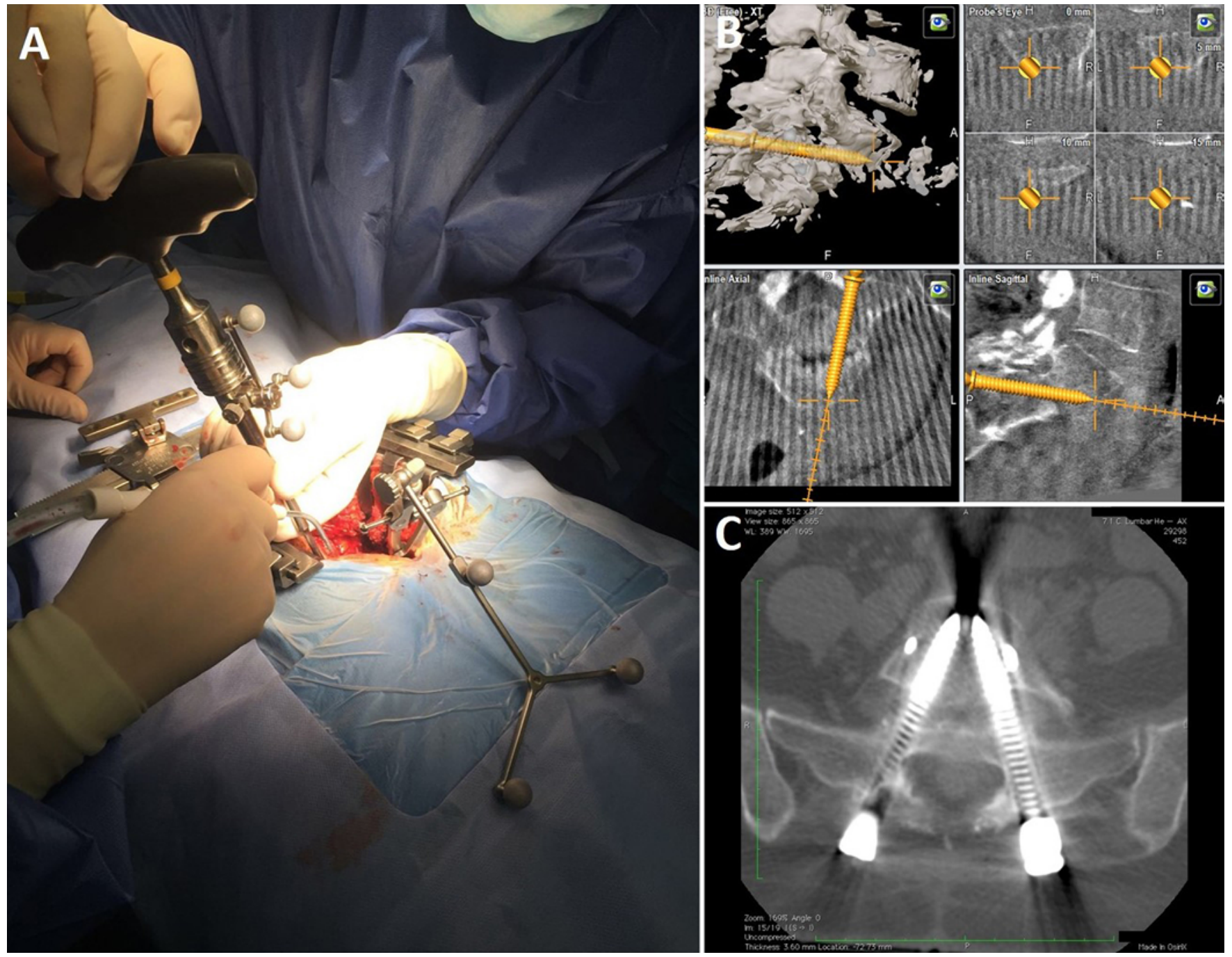

FIG. 3. A: Intraoperative photograph showing the use of a navigated awl-tap for transdiscal screw placement. B: Photograph of the navigation screen showing screw placement along the correct trajectory. C: Intraoperative axial CT image showing correctly positioned transdiscal screws.

was 235 minutes (range 180-280 minutes). There were no intraoperative complications.

The intraoperative CT performed at the end of the procedure showed correct position of the instrumentation in all cases, with no need for repositioning of the screws. Also, none of the patients required a transfusion after the procedure.

Low-back pain lasting longer than 2 years was the main symptom in all the cases, radiating to the lower extremities mainly at the pelvic girdle. The mean preoperative VAS score was 6.5 (range 5-9), and the mean preoperative ODI score was 49.2 (range 18-71.1). However, in 3 of the cases low-back pain was accompanied by radiculopathy to the feet.

The mean duration of follow-up was 16 months (range 9-24 months), and no complication was observed during this period. The mean postoperative VAS score was 4 (range 2-7), and the mean postoperative ODI score was 37.7 (range 4.4-64). There was a significant clinical im- provement postoperatively in both VAS (Wilcoxon signedrank test, $\mathrm{p}=0.01$ ) and ODI (Wilcoxon signed-rank test, $\mathrm{p}$ $=0.012$ ) scores. Also, none of the patients have returned to our pain unit because of back pain, and all but 2 patients have reduced their intake of pain medication. None of the patients had increased their analgesic dosage at the last follow-up. Six patients were starting exercise and 2 had returned to their jobs. There was no significant difference between preoperative and postoperative values for any of the spinopelvic parameters (Table 1). Review of follow-up images by the independent radiologist showed no pseudarthrosis or implant breakage or malplacement, and fusion was obtained in all cases (Fig. 4).

\section{Discussion}

HGS is a low-incidence pathology, which makes it difficult to standardize an approach for its treatment. Many techniques have been described for HGS treatment, 
TABLE 1. Summary of clinical and radiological data in 8 adult patients with HGS

\begin{tabular}{lccc}
\hline \multicolumn{1}{c}{ Variable } & Preop & Postop & p Value \\
\hline Clinical data & & & \\
\hline VAS, lumbar pain & $6.5(1.5)$ & $4(1.7)$ & 0.01 \\
\hline ODI & $49.2(19.4)$ & $37.7(22)$ & 0.012 \\
\hline Radiological data & & \\
\hline Slip angle & $83.5^{\circ}\left(16.5^{\circ}\right)$ & $69.3^{\circ}\left(14.9^{\circ}\right)$ & 0.14 \\
\hline Pelvic incidence & $80.6^{\circ}\left(10.2^{\circ}\right)$ & $84.6^{\circ}\left(9.5^{\circ}\right)$ & 0.71 \\
\hline Pelvic tilt & $27.6^{\circ}\left(7.3^{\circ}\right)$ & $25^{\circ}\left(8.6^{\circ}\right)$ & 0.06 \\
\hline Sacral slope & $54.6^{\circ}\left(6^{\circ}\right)$ & $56.4^{\circ}\left(6^{\circ}\right)$ & 0.27 \\
\hline
\end{tabular}

Data are presented as mean (SD).

including anterior, posterior, and circumferential approaches. Abdu et al. ${ }^{1}$ were the first authors to describe a posterior approach through a pedicular transdiscal screw fixation in HGS. These authors cited 4 main advantages to this approach: it uses the concept of 3-column fixation achieved from a single posterior approach; it has demonstrated in vivo implementation of superior biomechanical principles, as the triangular construct provides a superior fixation strength; it has better contact with cortical bone; and the use of longer screws and greater penetration provides additional fixation. Subsequent studies of biomechanical properties of the transdiscal approach have demonstrated improvement in resistance against shear forces at the displaced level compared with posterolateral fusion, ${ }^{4,8}$ stiffness 1.6-1.8 times higher than that achieved with traditional pedicle screw fixation, ${ }^{18}$ and equivalent fixation to that achieved with the combination of interbody fusion and pedicle screw fixation. ${ }^{23}$ Another advantage is that a posterior approach is used more frequently in spine surgeries and is more familiar for less-experienced surgeons. As Collados-Maestre et al. ${ }^{5}$ reported, it can be difficult to obtain an adequate position of a sacral screw; however, in our experience, this limitation is easily resolved when surgeons have experience in image guidance.

Reduction of listhesis is one of the most controversial topics in HGS management. The aim of reduction is to restore spinal anatomy, mainly recommended in patients with sagittal imbalance, and to lower the risk of pseudarthrosis. However, neurological complications are described in $25 \%-30 \%$ of cases..$^{10,15,19}$ In a recent review of HGS, mainly in the pediatric population, Longo et al. ${ }^{17}$ showed that there was no statistically significant difference in neurological complications in patients with reduction compared with those patients without reduction, but pseudarthrosis was more frequent in patients with in situ fixation $(17.8 \%$ vs $5.5 \%, p=0.004)$. In our series, we did not perform a reduction, as there was no sagittal imbalance in any patient. One of the limitations of Longo and colleagues' analysis is that most of the studies (5 of 8) were performed in children, in whom neurological complications were less frequent than in adults.

Again, a lack of studies on HGS with a substantial proportion of adult patients makes it difficult to generate a reliable standard recommendation. One of the largest series, published by Collados-Maestre et al., 5 compared treatment with pedicle screws and treatment with transdiscal fixation. In their study, 25 patients (age range 14-60 years) were treated with transdiscal fixation. Complications reported for that group of patients included infection (8\%), implant misplacement (12\%), and implant breakage (4\%). The authors did not find any case of pseudarthrosis in the patients treated with transdiscal fixation, despite the fact that pseudarthrosis is one of the most frequent complications associated with in situ fusion and fixation procedures. ${ }^{17,23,26}$

In previously published studies, the incidence of reported complications related to transdiscal fixation for the treatment of HGS is generally quite low; with the exception of 1 study, in which the incidence was $77.8 \%,{ }^{26}$ the in-
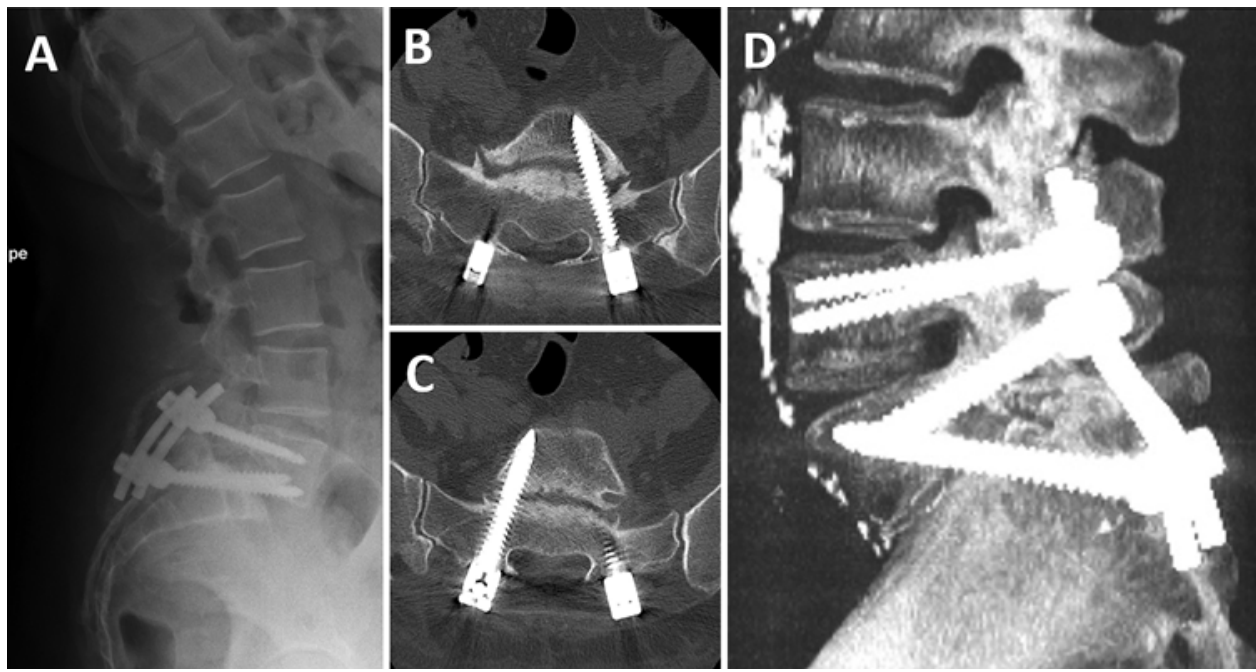

FIG. 4. Postoperative images. A: Lateral standing radiograph showing correct positioning of the instrumentation without signs of malplacement or pseudarthrosis. B and C: Axial CT images showing the proper placement of screws from the S-1 insertion point through the L-5 vertebral body. D: 3D CT reconstruction of L4-S1 transdiscal fixation. 
TABLE 2. Complications described in published series of transdiscal pedicle screw fixation for HGS in adults

\begin{tabular}{|c|c|c|c|c|c|c|c|c|}
\hline Authors \& Year & $\begin{array}{l}\text { No. of } \\
\text { Cases }\end{array}$ & $\begin{array}{l}\text { Overall } \\
\text { Morbidity }\end{array}$ & Pseudarthrosis & $\begin{array}{l}\text { Postop } \\
\text { Infection }\end{array}$ & $\begin{array}{c}\text { Intraop } \\
\text { Complication }\end{array}$ & $\begin{array}{l}\text { Neurological } \\
\text { Complication }\end{array}$ & $\begin{array}{c}\text { Screw } \\
\text { Malplacement }\end{array}$ & $\begin{array}{l}\text { Instrumentation } \\
\text { Failure }\end{array}$ \\
\hline Abdu et al., 1994 & 3 & & & & & & & \\
\hline Smith et al., 2001 & 9 & $77.8 \%$ & 2 & & 3 & 2 & & \\
\hline Boachie-Adjei et al., 2002 & 6 & $33.3 \%$ & & & 1 & 1 & & \\
\hline François et al., 2005 & 4 & & & & & & & \\
\hline Rodriguez-Olaverri et al., 2008 & 20 & $25 \%$ & 1 & 2 & 1 & & & 1 \\
\hline Lakshmanan et al., 2009 & 12 & $16.6 \%$ & & & 2 & & & \\
\hline Logroscino et al., 2012 & 15 & $13.3 \%$ & & 1 & & & 1 & \\
\hline Collados-Maestre et al., 2016 & 25 & $24 \%$ & & 2 & & & 3 & 1 \\
\hline Present study & 8 & $0 \%$ & & & & & & \\
\hline
\end{tabular}

Abdu et al. ${ }^{1}$ and François et al. ${ }^{8}$ did not state in their studies whether there were any complications. In our study, no complications were found at last follow-up.

cidence ranges from $13 \%{ }^{15}$ to $33 \%{ }^{4}$ (Table 2). The most frequent intraoperative complication described is dural tear, accounting for $17.8 \%$ of the reported complications. ${ }^{4,14,23,26}$ Other postoperative complications frequently described are infection (5\%), $, 5,23$ hardware failure (4.5\%), ${ }^{5,23}$ and implant malplacement (9\%), ${ }^{5,15}$ As Collados-Maestre et al. ${ }^{5}$ revealed in their review, the difficulty in placing the implant may lie mainly in reaching the L-5 vertebral body. Malplacement of hardware and its failure are both common complications of transdiscal fixation for the treatment of spondylolisthesis. ${ }^{15,23}$ Screw failure could also be related to increased forces due to malplacement. In these cases, online 3D image guidance offers a better approach, improving security in the moment of the implant position and decreasing the incidence of complications, as we have shown in our series. To our knowledge, the use of image guidance for transdiscal screws has only been previously reported in the literature a single time-by Beringer et al., ${ }^{3}$ who described an illustrative case of HGS treated through an anterior L4-5 ALIF (anterior lumbar interbody fusion) and transdiscal pedicle screw placement with good results. Our results presented here further demonstrate that screw malplacement and hardware breakage are greatly reduced with the use of online 3D image guidance compared with the results from previous reports. Nonetheless, the main limitation in our study is a short mean duration of followup, and this may have led to underestimation of implant failure and the presence of pseudarthrosis as well as the fusion rate in the most recent cases.

\section{Conclusions}

The use of transdiscal pedicle screws with in situ fusion is a good option for treating HGS in patients with good sagittal balance. This technique has some advantages compared with other approaches, including the increased familiarity of the surgical field for spine surgeons and the relatively low frequency of complications, which could be reduced using image-guidance systems, commonly used in most of the spine centers treating this kind of pathology.

\section{References}

1. Abdu WA, Wilber RG, Emery SE: Pedicular transvertebral screw fixation of the lumbosacral spine in spondylolisthesis. A new technique for stabilization. Spine (Phila Pa 1976) 19:710-715, 1994

2. Acosta FL Jr, Ames CP, Chou D: Operative management of adult high-grade lumbosacral spondylolisthesis. Neurosurg Clin N Am 18:249-254, 2007

3. Beringer WF, Mobasser JP, Karahalios D, Potts EA: Anterior transvertebral interbody cage with posterior transdiscal pedicle screw instrumentation for high-grade spondylolisthesis. Technical note. Neurosurg Focus 20(3):E7, 2006

4. Boachie-Adjei O, Do T, Rawlins BA: Partial lumbosacral kyphosis reduction, decompression, and posterior lumbosacral transfixation in high-grade isthmic spondylolisthesis: clinical and radiographic results in six patients. Spine (Phila Pa 1976) 27:E161-E168, 2002

5. Collados-Maestre I, Lizaur-Utrilla A, Bas-Hermida T, PastorFernandez E, Gil-Guillen V: Transdiscal screw versus pedicle screw fixation for high-grade L5-S1 isthmic spondylolisthesis in patients younger than 60 years: a case-control study. Eur Spine J 25:1806-1812, 2016

6. Dubousset J: Treatment of spondylolysis and spondylolisthesis in children and adolescents. Clin Orthop Relat Res (337):77-85, 1997

7. Fairbank JC, Pynsent PB: The Oswestry disability index. Spine (Phila Pa 1976) 25:2940-2952, 2000

8. François J, Lauweryns P, Fabry G: Treatment of high-grade spondylolisthesis by posterior lumbosacral transfixation with transdiscal screws: surgical technique and preliminary results in four cases. Acta Orthop Belg 71:334-341, 2005

9. Guyatt GH, Townsend M, Berman LB, Keller JL: A comparison of Likert and visual analogue scales for measuring change in function. J Chronic Dis 40:1129-1133, 1987

10. Hire JM, Jacobs JM, Bundy JV, DeVine JG: A modified Bohlman technique using a novel implant for treatment of high-grade spondylolisthesis. J Neurosurg Spine 22:80-83, 2015

11. Kalichman L, Kim DH, Li L, Guermazi A, Berkin V, Hunter DJ: Spondylolysis and spondylolisthesis: prevalence and association with low back pain in the adult community-based population. Spine (Phila Pa 1976) 34:199-205, 2009

12. Ko SB, Lee SW: Prevalence of spondylolysis and its relationship with low back pain in selected population. Clin Orthop Surg 3:34-38, 2011

13. Labelle H, Roussouly P, Berthonnaud E, Transfeldt E, O'Brien M, Chopin D, et al: Spondylolisthesis, pelvic incidence, and spinopelvic balance: a correlation study. Spine (Phila Pa 1976) 29:2049-2054, 2004

14. Lakshmanan P, Ahuja S, Lewis M, Howes J, Davies PR: 
Transsacral screw fixation for high-grade spondylolisthesis. Spine J 9:1024-1029, 2009

15. Logroscino CA, Tamburrelli FC, Scaramuzzo L, Schirò GR, Sessa S, Proietti L: Transdiscal L5-S1 screws for the treatment of adult spondylolisthesis. Eur Spine J 21 (Suppl 1):S128-S133, 2012

16. Lombardi F, Custodi VM, Pugliese R, Risso A, Gaetani P, Butti G: Treatment of high-grade spondylolisthesis with Schanz recoil screws: our experience. Eur Spine J 22 (Suppl 6):S914-S918, 2013

17. Longo UG, Loppini M, Romeo G, Maffulli N, Denaro V: Evidence-based surgical management of spondylolisthesis: reduction or arthrodesis in situ. J Bone Joint Surg Am 96:53-58, 2014

18. Minamide A, Akamaru T, Yoon ST, Tamaki T, Rhee JM, Hutton WC: Transdiscal L5-S1 screws for the fixation of isthmic spondylolisthesis: a biomechanical evaluation. J Spinal Disord Tech 16:144-149, 2003

19. Palejwala A, Fridley J, Jea A: Transsacral transdiscal L5-S1 screws for the management of high-grade spondylolisthesis in an adolescent. J Neurosurg Pediatr 17:645-650, 2016

20. Passias PG, Poorman CE, Yang S, Boniello AJ, Jalai CM, Worley N, et al: Surgical treatment strategies for high-grade spondylolisthesis: a systematic review. Int J Spine Surg 9:50, 2015

21. Ray CD: Threaded fusion cages for lumbar interbody fusions. An economic comparison with 360 degrees fusions. Spine (Phila Pa 1976) 22:681-685, 1997

22. Roche MB, Rowe GG: The incidence of separate neural arch and coincident bone variations; a survey of 4,200 skeletons. Anat Rec 109:233-252, 1951

23. Rodriguez-Olaverri JC, Zimick NC, Merola A, Vicente J, Rodriguez J, Tabuenca A, et al: Comparing the clinical and radiological outcomes of pedicular transvertebral screw fixation of the lumbosacral spine in spondylolisthesis versus unilateral transforaminal lumbar interbody fusion (TLIF) with posterior fixation using anterior cages. Spine (Phila Pa 1976) 33:1977-1981, 2008

24. Sarwahi V, Wendolowski SF, Gecelter RC, Amaral T, Lo Y, Wollowick AL, et al: Are we underestimating the significance of pedicle screw misplacement? Spine (Phila Pa 1976) 41:E548-E555, 2016

25. Smith BW, Joseph JR, Kirsch M, Strasser MO, Smith J, Park
P: Minimally invasive guidewireless, navigated pedicle screw placement: a technical report and case series. Neurosurg Focus (2):E9, 2017

26. Smith JA, Deviren V, Berven S, Kleinstueck F, Bradford DS: Clinical outcome of trans-sacral interbody fusion after partial reduction for high-grade L5-S1 spondylolisthesis. Spine (Phila Pa 1976) 26:2227-2234, 2001

27. Tian NF, Huang QS, Zhou P, Zhou Y, Wu RK, Lou Y, et al: Pedicle screw insertion accuracy with different assisted methods: a systematic review and meta-analysis of comparative studies. Eur Spine J 20:846-859, 2011

28. Tian W, Han XG, Liu B, Liu YJ, He D, Yuan Q, et al: Posterior reduction and monosegmental fusion with intraoperative three-dimensional navigation system in the treatment of high-grade developmental spondylolisthesis. Chin Med J (Engl) 128:865-870, 2015

\section{Disclosures}

The authors report no conflict of interest concerning the materials or methods used in this study or the findings specified in this paper.

\section{Author Contributions}

Conception and design: Delgado-Fernández, Pulido, GarcíaPallero, Sola. Acquisition of data: Delgado-Fernández, GarcíaPallero, Blasco, Frade-Porto. Analysis and interpretation of data: Delgado-Fernández, Pulido, García-Pallero, Blasco, Frade-Porto. Drafting the article: Delgado-Fernández, Pulido, García-Pallero, Frade-Porto. Critically revising the article: Delgado-Fernández, Pulido, García-Pallero, Blasco, Frade-Porto, Sola. Reviewed submitted version of manuscript: Pulido, Blasco, Sola. Approved the final version of the manuscript on behalf of all authors: DelgadoFernández. Statistical analysis: Delgado-Fernández, GarcíaPallero. Administrative/technical/material support: Sola. Study supervision: Sola.

\section{Correspondence}

Juan Delgado-Fernández: University Hospital La Princesa, Madrid, Spain. juan.delgado.fdez@gmail.com. 